Supporting Information for:

\title{
Mask-directed multiphoton lithography
}

\author{
Bryan Kaehr and Jason B. Shear
}

Department of Chemistry \& Biochemistry and the Institute for Cellular \& Molecular Biology, The University of Texas, Austin, TX 78712

\section{Supplemental movie captions}

S1. Video clip of Figure $2 \mathrm{C}$ image sequence showing smooth swimming E. coli enter the two-story microcontainer and proceed through the ground floor passageway to be shunted vertically into the second-floor chamber. Video rate is $3 \mathrm{X}$ real time.

S2. Real-time video clip showing a single entrapped bacterium 5 minutes after fabrication of plug to seal the chamber (Figure 3D, panel 2).

S3. Real-time video clip showing bacterium (Figure 3D, between panels 2 and 3) immediately before the first cell division.

S4. Real-time video clip showing cells after several divisions (ca. Figure 3D, panel 5).

\section{Materials}

Methylene blue (M-4159) and flavin adenine dinucleotide (FAD, F-6625) was supplied by Sigma-Aldrich (St. Louis, MO). Bovine serum albumin (BSA, BAH64-0100) was supplied by Equitech-Bio (Kerrville, TX). Avidin (A-887) and fluorescein biotin (B-1370) were supplied by Molecular Probes, (Eugene, OR). All chemicals and solvents were stored according to supplier's 
specifications and used without further purification. Office grade transparency film for laser printers was used to produce photomasks on an HP Laser Jet 2100TN.

\section{Strains}

E. coli strains RP437 (wild-type, wt) and RP9535 (smooth-swimming, $\Delta c h e A$ ), kindly provided by John S. Parkinson (Department of Biology, University of Utah), were grown aerobically in tryptone broth $\left(32{ }^{\circ} \mathrm{C}\right)$ and harvested at mid-log phase. Cells were diluted $20-100$ fold into PBS (10 mM potassium phosphate, $\mathrm{pH}$ 7.0) for experiments with fabricated microchambers.

\section{Matrix fabrication}

Matrixes composed of photo-crosslinked protein were fabricated onto untreated \#1 microscope cover glass using the output of a mode-locked titanium:sapphire laser (Tsunami; Spectra Physics, Mountain View, CA) operating at 730 to $740 \mathrm{~nm}$. The laser beam was raster scanned into rectangular patterns using a confocal scanner (BioRad MRC600) and brought to focus between the scanbox and the microscope. Placing masks in this focal plane (referred to in the text as the 'mask plane') allowed the greatest fidelity in the fabricated object since the mask plane is conjugate with the microscope specimen plane, although masks could be used (with less edge resolution) when placed at any position between the scanbox and the microscope $(18 \mathrm{~cm})$. For example, the Texas-shaped micro-gradient in Figure 1B was fabricated using two masks simultaneously: a negative photo mask used to define the gradient edges was placed in the mask plane while a 
second, straight-edged fully opaque mask was translated during fabrication approximately $7.5 \mathrm{~cm}$ outside of the mask plane. Masks were aligned manually by adjusting the $X Y$ position of the mask during test photofabrication procedures. Moving masks were generally translated at a linear velocity of 100 to $200 \mu \mathrm{m} \mathrm{s}^{-1}$ using rectangular scan frequencies (the inverse of the time to complete a raster-scanned rectangle) of $3 \mathrm{~Hz}$.

The laser output was adjusted to approximately fill the back aperture of an oil-immersion objective (Zeiss 100x Fluar, 1.3 numerical aperture) situated on a Zeiss Axiovert inverted microscope system. Desired powers (30-40 $\mathrm{mW}$ before the back aperture of the microscope objective) were obtained by attenuating the laser beam using a half-wave plate/polarizing beam splitter pair. To extend structures along the $z$ dimension (i.e., along the optical axis), the position of the laser focus was translated manually within fabrication solutions using the microscope fine focus adjustment. By removing the mask once the desired structure height was attained, microchambers could be readily sealed from the top with closed rectangular roofs. Typical microchambers having heights of $2-10 \mu \mathrm{m}$ were produced by allowing two full scans to be rastered across the sample per micron of vertical travel. This procedure allows fully formed $3 \mathrm{D}$ objects to be fabricated on time scales of $10-30$ seconds.

Microstructures composed of photo-cross-linked BSA were fabricated from solutions containing protein at $320-400 \mathrm{mg} \mathrm{mL}^{-1}$ and $2-3 \mathrm{mM}$ methylene blue as a photosensitizer. For biocompatible fabrication (e.g., Figure 3), flavin adenine dinucleotide ( $5 \mathrm{mM}$ ) was used as the photosensitizer. 
The practical (lateral) resolution that could be achieved for microstructures in these studies, $\sim 0.5 \mu \mathrm{m}$, was lower than we have achieved in some previous instances for protein photocrosslinking (see reference 4, main text), a result of the mask quality, the speed at which structures were fabricated, and, in the case of the SEM images, the preparation process for imaging. As typical for high-numerical aperture multiphoton excitation, the voxel is somewhat elongated in the vertical dimension (reference 1 in the main text). When microstructure fabrication required focusing vertically through a significant thickness of previously photocrosslinked protein (e.g. the two-story structure in Figure 2), resolution was diminished further. Heterogeneity in protein thickness for microstructures shown in Figure 2 and the Supporting Figure is likely the result of artifacts in the scanning process, as they also are observed in some cases where no mask is used.

\section{Cell incubation in BSA microchambers}

After fabricating the protein plug to trap a single bacterium in a microchamber (Fig 3D panel 2; chamber dimensions, $10 \times 10 \times 4 \mu \mathrm{m}$ ), the cell was incubated at ambient temperature $\left(22^{\circ} \mathrm{C}\right)$ in tryptone broth in $1 \mathrm{~mL}$ dish. The media was replaced at approximately 6-hour intervals and the microchamber was monitored over a period of 3 days.

\section{Fluorescence microscopy}

Wide-field fluorescence imaging was performed on the Axiovert microscope, which was equipped with a mercury-arc lamp and standard "red" and "green" filter sets (Chroma, Rockingham, VT). Fluorescence emission was collected 
using the Fluar 100x objective and detected using a 12-bit $1392 \times 1040$ element CCD (Cool Snap HQ; Photometrics, Tucson, AZ). Data were processed using Image $\mathrm{J}$ and Metamorph (Universal Imaging, Sunnyvale, CA) image-analysis software.

\section{Scanning electron microscopy (SEM) preparation}

Samples were fixed in $3.5 \%$ gluteraldehyde solution for $20 \mathrm{~min}$ and dehydrated by using 10-min sequential washes (2:1 ethanol/ $\mathrm{H}_{2} \mathrm{O}$; twice in $100 \%$ ethanol; $1: 1$ ethanol/methanol; $100 \%$ methanol; all solutions stated as $v / v)$, allowed to air-dry for $3 \mathrm{~h}$, and sputter-coated to a nominal thickness of $12-15 \mathrm{~nm}$ with $\mathrm{Au} / \mathrm{Pd}$.
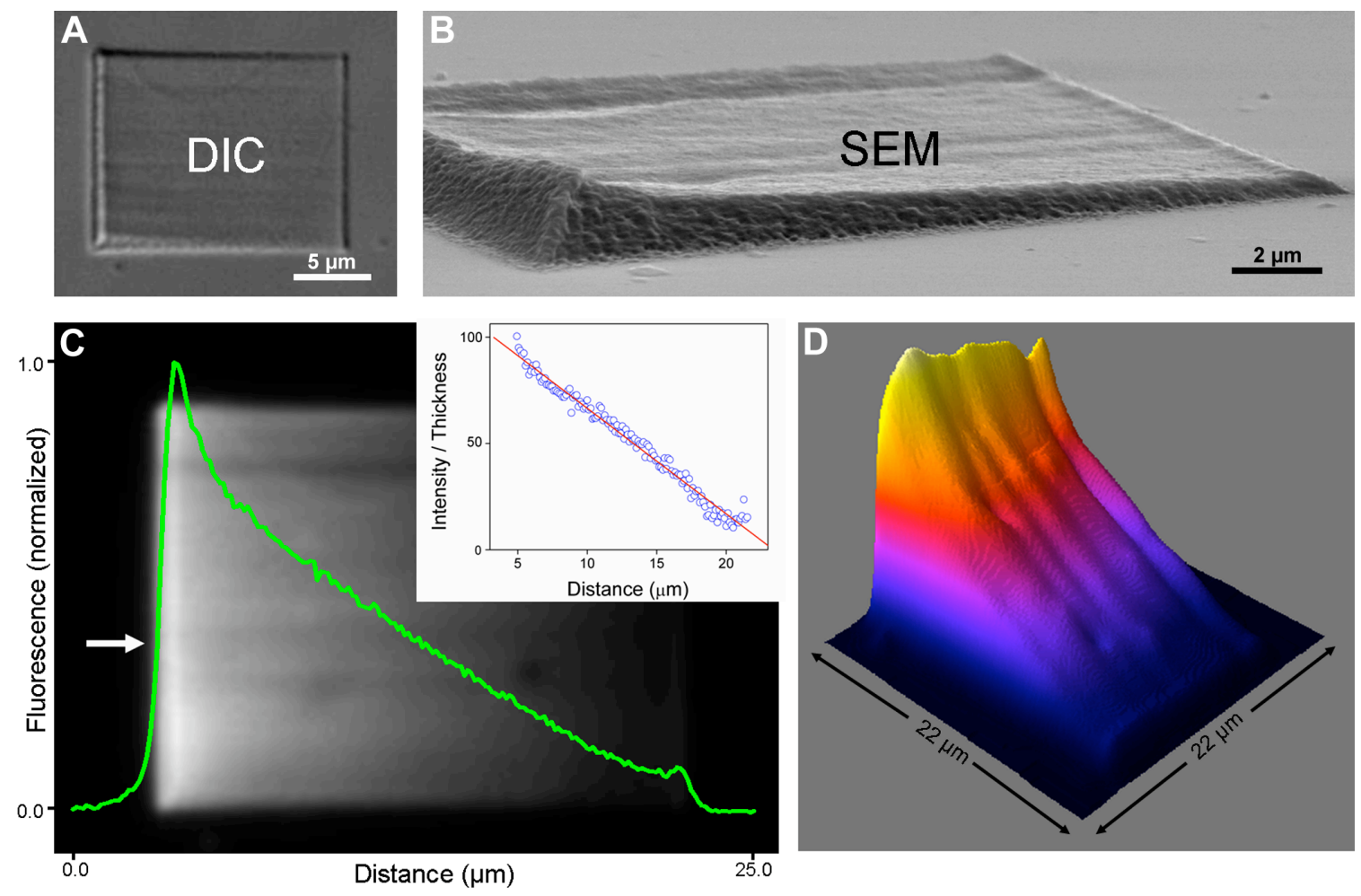
Supporting Figure 1 (Fig S1): Use of a moving mask to create a gradient in both thickness and chemical functionalization across a protein microstructure. A gradient microstructure was fabricated from a solution containing $90 \%$ BSA and $10 \%$ avidin (wt/wt; total protein concentration was $320 \mathrm{mg} \mathrm{mL}^{-1}$ ) and methylene blue ( $3 \mathrm{mM}$ ). During laser scanning, a fully opaque straight-edge mask was translated such that its image in the fabrication plane was swept at a rate of $2 \mu \mathrm{m} \mathrm{s}^{-1}$. The resultant BSA/avidin microstructure was incubated in $2 \mu \mathrm{M}$ fluorescein biotin for $10 \mathrm{~min}$, rinsed 10 times in PBS ( $\mathrm{pH} 7.0$ ), and imaged via fluorescence. (A, B) DIC and SEM microscopy reveal that changes in laser exposure times across the protein structure cause a thickness gradient. (C) Plot (green line) representing the fluorescence intensity of a horizontal line drawn across the structure (from arrow). This intensity was divided by the thickness of the structure (inset) to yield the functional gradient density (i.e., normalized for structure thickness). From this data, the fluorescence intensity gradient is shown to be a convolution of structure thickness and functional density (i.e., biotinbinding capacity of avidin). Panel $D$ is a 3D surface intensity plot of the fluorescent image in panel $\mathrm{C}$ and shows that the gradient is maintained across the surface of the microstructure. 